OPEN ACCESS

Edited by:

Kee-Hong Choi,

Korea University, South Korea

Reviewed by:

Dong Gi Seo,

Hallym University, South Korea

Sooyeon Aly Suh,

Sungshin Women's University,

South Korea

*Correspondence:

Xiaofang Yu

494975955@qq.com

Yan Cai

cy1979123@aliyun.com

Dongbo Tu

tudongbo@aliyun.com

Specialty section:

This article was submitted to Quantitative Psychology and

Measurement,

a section of the journa

Frontiers in Psychology

Received: 05 March 2021 Accepted: 28 April 2021

Published: 28 May 2021

Citation:

Quan J, Yu X, Cai Y and Tu D (2021) Comparison of Psychometric

Characteristics for Five Versions of the Interpersonal Needs Questionnaire in

Teenagers Sample.

Front. Psychol. 12:676361

doi: 10.3389/fpsyg.2021.676361

\section{Comparison of Psychometric Characteristics for Five Versions of the Interpersonal Needs Questionnaire in Teenagers Sample}

\author{
Jiaxin Quan ${ }^{1}$, Xiaofang $\mathrm{Yu}^{2 *}$, Yan $\mathrm{Cai}^{{ }^{1 *}}$ and Dongbo Tu${ }^{1 *}$ \\ ${ }^{1}$ School of Psychology, Jiangxi Normal University, Nanchang, China, ${ }^{2}$ Center of Mental Health Education and Research, \\ School of Psychology, Jiangxi Normal University, Nanchang, China
}

Interpersonal Needs Questionnaire (INQ) is a self-report measure of perceived burdensomeness and thwarted belongingness with five versions in recent studies. There are five versions of INQ. But results from studies using different versions are quite different. Current suicide behavior among teenagers has attracted much attention. But which version is more suitable for teenage samples is still uncertain. It is important to compare the potential differences in different versions of INQ to identify the most psychometrically available version to predict teenagers' acquired capability for suicide and provide them with timely help to reduce teenagers' suicide rates. This study compared the construct validity, internal consistency, validity, and average test information of each version in the sample of teenagers. Results showed the 10-item version provided the most average test information in both thwarted belongingness subscale and perceived burdensomeness subscale, and the INQ-10 is more suitable for teenage samples.

\footnotetext{
Keywords: teenagers, item response theory, comparison, psychometric characteristics, interpersonal needs questionnaire
}

\section{INTRODUCTION}

Suicide is a major social and public health problem facing the world. Nearly 800,000 people commit suicide each year, and the number of suicide attempts is many times the number of suicides. Suicide occurred throughout the lifespan and it was the third leading cause of death in 15-19-yearold worldwide in 2016 (World Health Organization, 2019). In the general population, attempted suicide is the biggest risk factor for suicide. Suicide attempts peak in mid-puberty (Carballo et al., 2019). Suicide and serious self-harm not only seriously endanger the lives and health of young people, but also cause serious losses to individuals, families, and society.

The interpersonal-psychological theory of suicide (IPTS) was first proposed by Joiner (2005) and further expanded by Van Orden et al. (2010). This theory surpasses the previous theories of suicide in that it explains why the vast majority of people with suicidal ideation do not attempt suicide. IPTS proposes that suicidal behavior occurs only when an individual has both the desire to die and the acquired capability to engage in suicidal behavior. The desire to die is an individual's desire to end her/his life, which roughly corresponds to the common definition of suicidal ideation (Van Orden et al., 2008a). The acquired ability to engage in suicidal behavior is a learned ability, which means that through repeated exposure to painful and provocative events, the fear of death can be reduced and the tolerance of physical pain can be enhanced. According to the prediction of 
IPTS (Joiner, 2005; Van Orden et al., 2010), whether an individual has suicidal ideation depends on whether belongingness of the individual is met (thwarted belongingness, TB) and whether the individual considers himself/herself a burden to others (perceived burdensomeness, $\mathrm{PB}$ ), and suicidal ideation will not turn into suicidal behavior until the acquired capability is large enough. Therefore, the interpersonal-psychological theory of suicide is defined as the framework of ideation-to-action (Klonsky and May, 2014; Klonsky et al., 2016).

Since the interpersonal-psychological theory of suicide was proposed, it has inspired many empirical studies on the causes of suicidal ideation, attempts, and fatalities. Research on the interpersonal-psychological theory of suicide has been conducted in different samples, such as undergraduates (Hagan et al., 2015; Suh et al., 2017), prison inmates (Mandracchia and Smith, 2015), physicians (Fink-Miller, 2015), older adults (Cukrowicz et al., 2013), psychiatric inpatients and outpatients (Monteith et al., 2013), military service members (Bryan et al., 2010), sexual minorities (Silva et al., 2015), and firefighters (Chu et al., 2016). The interpersonal-psychological theory of suicide was also validated cross-culturally across Korean and US undergraduate students (Suh et al., 2017). Moreover, based on IPTS, Van Orden (2009) further confirmed and extended the theory that perceived burdensomeness and thwarted belongingness were combined into interpersonal needs and constructed a corresponding Interpersonal Needs Questionnaire (INQ) with 25 items to reflect whether current interpersonal relationship needs of the individual were met. On account of the multicollinearity between thwarted belongingness and perceived burdensomeness, the 12-item INQ was later developed (Van Orden et al., 2008a). And the original authors proposed a 15item version (Van Orden et al., 2012). An 18-item version was validated in a book on the interpersonal theory (Joiner et al., 2009) and a 10-item INQ was validated for use in military samples (Bryan et al., 2010). Each of the shorter versions of INQ is a subset of the original 25 -item version. The 18 -item version has been used primarily in the older adult, veterans, and college student samples in the US and college student samples in China (e.g., Davidson et al., 2011; Rasmussen and Wingate, 2011; Wong et al., 2011; Monteith et al., 2013; Zhang et al., 2013; Suh et al., 2017). The 15 -item version introduced as an empirically derived refinement of the INQ-25 has been used in college student samples in the US, Singapore, China, and Switzerland (INQ-15; e.g., Van Orden et al., 2012; Hill and Pettit, 2013; Li et al., 2015; Baertschi et al., 2017; Teo et al., 2018), and Hallensleben et al. (2016) administrated the INQ-15 to a sample of German general population aged 14-75 years. The 12-item version has been used in a variety of samples in the US (e.g., Van Orden et al., 2008a; Davidson et al., 2009; Freedenthal et al., 2011; Hill and Pettit, 2012; Lamis and Lester, 2012). The 10-item version has been used primarily in military samples (e.g., Bryan et al., 2010, 2012, 2013; Bryan, 2011). According to previous studies (e.g., Bryan, 2011; Davidson et al., 2011; Freedenthal et al., 2011; Baertschi et al., 2017), the original version and its four shorter versions had acceptable internal consistencies and validities.

Although previous studies showed the INQ predicted suicide behaviors significantly (e.g., Van Orden et al., 2008a,b), a large number of discrepant studies found different versions of INQ had some differences in predicting suicide behaviors. For example, the INQ-25 (Anestis and Joiner, 2011), the INQ-18 (Wong et al., 2011), the INQ-12 (Hill and Pettit, 2012), and INQ-10 (Bryan et al., 2012) have been confirmed that perceived burdensomeness was a significant predictor, but thwarted belongingness was not. On the contrary, both perceived burdensomeness and thwarted belongingness had adequate predictive validity in the 12 -item version (Lamis and Malone, 2011) and the 15-item version (Van Orden et al., 2012). The differences in the predictive validity of the measures confuse future researchers on which version of INQ should be used. Furthermore, there is documentation on how to select items in the previous literature for the INQ-25 and INQ-15. But for the other versions, it is unclear how to select items from the original 25-item. Therefore, it is necessary to investigate and compare the psychometric characteristics of the five versions of the INQ in the same sample.

In the past, most psychological constructs of self-rating measurements have been assessed through classical test theory (CTT), which focuses on construct validity, internal consistency, and test-retest stability (Hunsley and Mash, 2008). However, CTT methods to assess interpersonal needs of an individual rely on the total score or transformed total score and fail to offer individuals with more direct information about his/her interpersonal needs range. This goal can be realized through the application of the item response theory (IRT). As the basis of the latest psychometric techniques, the IRT methods can provide analyses of individual latent traits (e.g., interpersonal needs) and item characteristics.

Based on IRT, item and test-information functions can be calculated by integrating the estimated parameters in IRT models to describe graphically and most precisely evaluate the regions of the individual latent trait continuum. Based on the IRT, item and test-information functions assessed on the same latent trait instrument are comparable in different measurements (Fayers, 2004). Therefore, based on the IRT methods, multiple inventories on a single and common metric can be comparable. What is more, the IRT methods can provide suggestions on which item or inventory can provide the most information for different latent traits (Olino et al., 2012).

Until now, no study has compared the psychometric characteristics of different INQ versions based on the IRT in the teenage samples and no study has investigated which version of INQ is more suitable for teenage samples. But the issue of teenagers' suicide cannot be ignored. From the Centers for Disease Control and Prevention, the incidence of suicide attempts peaks in mid-puberty, and the suicide mortality rate steadily increases throughout the teenage period with age. It is the third leading cause of death among young people aged 1024 (Centers for Disease Control Prevention, 2017). The suicidal characteristics of teenagers are different from those of adults (Parellada et al., 2008). Hence, an effective tool is needed to assess the ranges of interpersonal needs of young people to identify a higher risk of suicidal behaviors. Predicting which individual is likely to commit suicide will help establish strategies for youth suicide prevention and intervention. It is important to explore the potential differences in different versions of INQ 
to identify the most psychometrically available version to assess the range of interpersonal needs. Moreover, results from many empirical studies found that the relationship between thwarted belongingness and suicidal behaviors was generally weaker in comparison to perceived burdensomeness (Ma et al., 2016; Chu et al., 2017). It is necessary to verify whether this phenomenon exists in teenagers.

In this study, we have investigated and compared the psychometric properties of five versions of the INQ based on an IRT model, to identify the version (or versions), which is more suitable for teenage samples to assess interpersonal needs. Since INQ-15 is a refinement of the INQ-25 (see Van Orden et al., 2012) and how the other versions select items from the original 25-item version is unknown, we hypothesized that the 15-item version would have adequate psychometric characteristics concerning factor structure, internal consistency, and validity. According to Hill et al. (2015), we hypothesized that the 15 -item version and 10 -item version would show more satisfactory psychometric characteristics compared to the other versions. Based on the IPTS and previous studies, we also hypothesized that in the INQ-12, INQ-18, and INQ-25, perceived burdensomeness, but not thwarted belongingness, would significantly predict capability for suicide and that both $\mathrm{PB}$ and TB would significantly predict capability for suicide in the INQ-15 and the INQ-10. Since no research were testing average test information and the differential item functioning caused by gender in the five versions, no specific hypotheses were made. The software $\mathrm{R}$ (Version 3.3.21) and the $\mathrm{R}$ packages mirt (Version 1.24; Chalmers, 2012) were employed to estimate item parameters. Moreover, we also compared which version could provide greater average test information in a larger range of latent traits. What is more, there was a conversion table provided to obtain the transformed scores of each version. At last, this study is also exempted to test the hypotheses of the IPTS and guide refinement of the IPTS.

\section{METHODS}

\section{Participants}

The complete data in the study was available for 905 individuals after deleting the missing response data. Participants were Chinese teenagers from four middle schools in two provinces of China. The mean age of the participants was 15.03 years ranged from 12 to 18 years $(S D=1.70)$. Participants were predominantly male $(60.4 \%)$, only child $(77.8 \%)$, and urban (78.6\%). Participants were from six grades: Junior One (10.2\%), Junior Two (22.9\%), Junior Three (10.5\%), Senior One (16.6\%), Senior Two (16.8\%), and Senior Three (23.1\%). Both written and verbal consents were acquired from parents of the participants before taking part in the experiment. This study was approved by the Ethics Committee of Jiangxi Normal University and was conducted following the ethical principles of the Declaration of Helsinki.

\section{Measures}

The Interpersonal Needs Questionnaire (INQ; Van Orden, 2009) is a 25-item self-report measure used to assess thwarted belongingness and perceived burdensomeness. Each of the 18-, $15-, 12-$, and 10-item versions is a subset of the original 25 -item version (see Table 1). Each item is rated on a 7-point Likert scale ranging from 1 (not at all true for me) to 7 (very true for me). The higher scores represent thwarted belongingness and perceived burdensomeness of the heavier individuals. The coefficients of Cronbach's alpha of five versions ranged from 0.91 to 0.95 in the current study.

The revised UCLA Loneliness Scale (Russell et al., 1980) is a 20-item self-report measure of loneliness. Participants are asked to rate the frequency of satisfaction and dissatisfaction with social relationships. All items are on a 4-point Likert scale ranging from 1 (never) to 4 (always). The higher scores represent higher levels of loneliness. Russell et al. (1980) reported a high internal consistency for the scale (Cronbach's alpha $=0.94)$, as well as support for the validity of the scale. In the current study, the scale had a high internal consistency (Cronbach's alpha $=0.91$ ).

The Perceived Social Support Scale (PSSS; Blumenthal et al., 1987) is a 12-item self-report measure of social support. Participants rate their degree of agreement to statements describing people can give them support on a 7-point Likert scale, ranging from "strongly agree" to "strongly disagree." The higher scores represent more social support. Blumenthal et al. (1987) reported a good internal consistency (Cronbach's alpha $=0.88$ ). In the current study, the scale had a high internal consistency (Cronbach's alpha $=0.94$ ).

The Acquired Capability for Suicide Scale-Chinese Version (ACSS-CV; Yang et al., 2019) is a 14-item self-report measure of acquired capability for suicide. Participants rate their degree of agreement to statements that describe their fearlessness about lethal self-injury on a 5-point Likert scale, ranging from "I do not agree at all" to "I fully agree." The higher scores represent less fearlessness about lethal self-injury. Yang et al. (2019) reported a proper internal consistency (Cronbach's alpha $=0.78$ ). In this study, the scale had a good internal consistency (Cronbach's alpha $=0.80)$.

\section{Analysis \\ Construct validity}

Confirmatory factor analysis (CFA) was used to examine the fit of the structure of five versions of the INQ, and several global fit indices were used to evaluate the fitness, including the root mean square error of approximation (RMSEA), the standardized root mean square residual (SRMR), the comparative fit index (CFI), and the Tucker-Lewis index (TLI). RMSEA and SRMR values < 0.08 (Browne and Cudeck, 1992; Hu and Bentler, 1999), and CFI and TLI values close to 0.95 or greater were considered adequate (Brown, 2006).

\section{Internal consistency and validity}

Cronbach's alpha coefficients were used to examine the internal consistency of each version scale and its subscales. And regression equations were constructed to test whether the five versions of $\mathrm{PB}$ and $\mathrm{TB}$ would predict acquired capability for suicide (measured by the ACSS-CV) significantly. 
TABLE 1 | Items included in each version of the INQ.

\begin{tabular}{|c|c|c|c|c|c|}
\hline Item & INQ-25 & INQ-18 & INQ-15 & INQ-12 & INQ-10 \\
\hline \multicolumn{6}{|l|}{ Perceived burdensomeness (PB) items } \\
\hline 1. The people in my life would be better off if I were gone & $x$ & $x$ & $x$ & $x$ & $x$ \\
\hline 2. I think I give back to society & $x$ & & & & \\
\hline 3. The people in my life would be happier without me & $x$ & $x$ & $x$ & $x$ & $x$ \\
\hline 4. I think I have failed the people in my life & $x$ & $x$ & & $x$ & \\
\hline 7. I think I am an asset to the people in my life & $x$ & & & & \\
\hline 8. I think my ideas, skills, or energy make a difference & $x$ & & & & \\
\hline 9. I think my death would be a relief to the people in my life & $x$ & & $x$ & & $x$ \\
\hline 10. I think I contribute to the well-being of the people in my life & $x$ & $x$ & & $x$ & \\
\hline 11. I feel like a burden on the people in my life & $x$ & $x$ & & $x$ & \\
\hline 15. I think I matter to the people in my life & $x$ & $x$ & & & \\
\hline \multicolumn{6}{|l|}{ Thwarted belongingness (TB) items } \\
\hline 16. Other people care about me & $x$ & $x$ & $x$ & $x$ & \\
\hline 17. I feel like I belong & $x$ & $x$ & $x$ & & $x$ \\
\hline 18. I rarely interact with people who care about me & $x$ & $x$ & $x$ & & \\
\hline 19. I am fortunate to have many caring and supportive friends & $x$ & $x$ & $x$ & & $x$ \\
\hline 20. I feel disconnected from other people & $x$ & $x$ & $x$ & $x$ & $x$ \\
\hline 21. I often feel like an outsider in social gatherings & $x$ & $x$ & $x$ & & $x$ \\
\hline 22. I feel that there are people I can turn to in times of need & $x$ & $x$ & $x$ & $x$ & \\
\hline 23. I feel unwelcome in most social situations & $x$ & & & & \\
\hline
\end{tabular}

INQ, Interpersonal Needs Questionnaire; INQ-25, 25-item version of INQ; INQ-18, 18-item version of INQ; INQ-15, 15-item version of INQ; INQ-12, 12-item version of INQ; INQ-10, 10-item version of INQ; $X$ represents an item included in a version.

\section{Differential item functioning}

If respondents from different groups (e.g., gender) with the same ability or proficiency level have different probabilities of choosing the same option for a certain item, then the item is flagged for differential item functioning (DIF; Kim, 2001). In the study, DIF analysis was used to identify systematic bias caused by gender. The IRTPRO program was used to calculate the DIF analysis based on the IRT method. This program performed DIF analysis according to Lord's IRT parameter comparison technique (Lord, 1977) under the framework of IRT.

\section{Average test information}

Test information is the sum of the information of each item. When the test provides more information to the participants with a certain potential trait value $(\theta)$, the standard error of the measurement of these participants will be smaller. In other words, the measurement will be more accurate. Based on the IRT model, we calculated the total test information curve of each subscale separately. The average test information was the total test information divided by the corresponding test length. The equation of item and test information in the graded response model (GRM; Samejima, 1969) are given, respectively, as

$$
I_{j}(\theta)=\sum_{t=0}^{m f_{j}} D^{2} a_{j}^{2}\left(P_{j t}^{*}-P_{j, t+1}^{*}\right)\left(1-P_{j t}^{*}-P_{j, t+1}^{*}\right)^{2},
$$

and

$$
I(\theta)=\sum_{j=1}^{n} I_{j}(\theta)
$$

where

$$
P_{j t}^{*}=\frac{1}{1+e^{-D a_{j}\left(\theta_{i}-b_{j t}\right)}} .
$$

Here $a_{j}$ and $b_{j t}$ denote the discrimination parameter and the location parameter of the item $j$ in GRM, respectively. $b_{j t}$ is the $t$ th location parameter for item $j$, which satisfies $b_{j 1}<b_{j 2}<\cdots$ $\cdot<b_{j m f_{j}}$; $m f_{j}$ represents the maximum score of item $j . \theta_{i}$ refers to the potential trait value of the participant $i . P_{j t}^{*}$ denotes the 
TABLE 2 | Global fit indices and correlations between subscales.

\begin{tabular}{|c|c|c|c|c|c|c|c|}
\hline Version & $\chi^{2}$ & $d f$ & RMSEA & CFI & TLI & SRMR & Correlation of PB and TB \\
\hline INQ-25 & 1751.38 & 272 & 0.08 & 0.89 & 0.88 & 0.05 & $0.77^{\star \star}$ \\
\hline INQ-18 & 718.39 & 132 & 0.07 & 0.94 & 0.93 & 0.05 & $0.72^{\star \star}$ \\
\hline INQ-15 & 347.15 & 87 & 0.06 & 0.96 & 0.96 & 0.04 & $0.70^{\star \star}$ \\
\hline INQ-12 & 240.63 & 52 & 0.06 & 0.97 & 0.96 & 0.04 & $0.66^{* *}$ \\
\hline INQ-10 & 146.71 & 32 & 0.06 & 0.98 & 0.97 & 0.03 & $0.70^{\star \star}$ \\
\hline
\end{tabular}

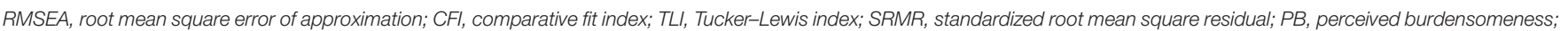
$T B$, thwarted belongingness. Bold values indicate acceptable model fit. ${ }^{* *} p<0.01$; same as below.

cumulative probability of participants $i$ gaining at least a score point $t$ on the item $j . D$ is a constant with a value of $1.7, I_{j}(\theta)$ refers to the information provided by item $j$ to participants whose potential trait value is $\theta, \mathrm{n}$ is the test length. $I(\theta)$ denotes the total test information.

\section{Expected Scores Conversion}

Based on the graded response model (GRM), we estimated the item parameters and transferred the potential trait value $(\theta)$ to calculate the expected scores of five versions of INQ, and then created a conversion table to implement a comparable process. To calculate the expected scores of subscales, the individual's response probability was calculated based on the GRM. The expected scores for $\theta_{i}$ can be calculated as

$$
\begin{gathered}
\text { Expected scores }\left(\theta_{i}\right)=\sum_{j=1}^{n} \sum_{t=1}^{m f_{j}} P_{j t}\left(\theta_{i}\right) \times t \\
P_{j t}\left(\theta_{i}\right)=P_{j t}^{*}-P_{j, t+1}^{*},
\end{gathered}
$$

where $P_{j t}\left(\theta_{i}\right)$ is the probability of getting $t$ score.

\section{RESULT}

\section{Confirmatory Factor Analyses}

Consistent with the literature to date, items of each of the five versions were loaded in two dimensions of perceived burdensomeness and thwarted belongingness and were set to load on their hypothesized factor with a correlation between the two factors. Similar to the work of Van Orden et al. (2012), and due to consistently high modification indices across different versions, each subscale had a pair of correlated residuals (items 1 and 3 on the perceived burdensomeness subscales and items 20 and 21 on the thwarted belongingness subscales). Since INQ-12 didn't include both item 20 and item 21, this pair of residuals was not included in the corresponding thwarted belongingness subscale.

Fit indices for the models are presented in Table 2. As can be seen in Table 2, the INQ-25 and the INQ-18 met few criteria for acceptable model fit, which was consistent with Hill et al. (2015), while the INQ-15, the INQ-12, and the INQ-10 met criteria for acceptable fit for all global indices of fit. These results indicated

\begin{tabular}{|c|c|c|c|c|}
\hline \multirow[t]{2}{*}{ Version } & \multirow{2}{*}{$\begin{array}{c}\text { Internal } \\
\text { consistency } \\
\text { (Cronbach's alpha) }\end{array}$} & \multicolumn{3}{|c|}{ Criterion validity } \\
\hline & & UCLA loneliness & PSSS & ACSS-CV \\
\hline INQ-25 & 0.95 & $0.75^{\star \star}$ & $-0.76^{\star \star}$ & $0.16^{\star *}$ \\
\hline PB & 0.93 & $0.66^{\star \star}$ & $-0.66^{\star *}$ & $0.13^{\star \star}$ \\
\hline TB & 0.89 & $0.80^{\star \star}$ & $-0.80^{\star \star}$ & $0.17^{\star \star}$ \\
\hline INQ-18 & 0.93 & $0.77^{\star \star}$ & $-0.76^{\star \star}$ & $0.15^{\star \star}$ \\
\hline PB & 0.91 & $0.65^{\star \star}$ & $-0.63^{\star \star}$ & $0.12^{* \star}$ \\
\hline TB & 0.87 & $0.78^{\star \star}$ & $-0.79^{\star \star}$ & $0.17^{\star \star}$ \\
\hline INQ-15 & 0.92 & $0.78^{\star *}$ & $-0.78^{\star \star}$ & $0.18^{\star *}$ \\
\hline PB & 0.90 & $0.64^{\star \star}$ & $-0.62^{\star \star}$ & $0.16^{\star \star}$ \\
\hline TB & 0.87 & $0.78^{\star \star}$ & $-0.79^{\star *}$ & $0.17^{\star \star}$ \\
\hline INQ-12 & 0.91 & $0.76^{\star \star}$ & $-0.75^{\star \star}$ & $0.15^{\star \star}$ \\
\hline PB & 0.90 & $0.66^{\star \star}$ & $-0.64^{\star \star}$ & $0.13^{\star *}$ \\
\hline TB & 0.81 & $0.75^{\star \star}$ & $-0.76^{\star \star}$ & $0.15^{\star \star}$ \\
\hline INQ-10 & 0.91 & $0.76^{\star \star}$ & $-0.74^{\star \star}$ & $0.19^{\star *}$ \\
\hline PB & 0.90 & $0.65^{\star \star}$ & $-0.62^{\star \star}$ & $0.17^{\star \star}$ \\
\hline TB & 0.81 & $0.76^{\star \star}$ & $-0.75^{\star \star}$ & $0.18^{\star \star}$ \\
\hline
\end{tabular}

TABLE 3 | Internal consistencies, and correlations between scales.

PSSS, Perceived Social Support Scale; ACSS-CV, Acquired Capability for Suicide ScaleChinese Version. ${ }^{* *} p<0.01$.

the INQ-15, the INQ-12 and the INQ-10 were more suitable in teenage samples.

\section{Internal Consistency and Validity}

To examine the internal consistency and criterion validity of each subscale, Cronbach's coefficient alphas and correlation coefficients were generated for each subscale (see Table 3). Both perceived burdensomeness and thwarted belongingness subscales were demonstrated good internal consistency (Cronbach's alphas ranged from 0.81 to 0.93 ). The internal consistency coefficients of thwarted belongingness subscales were smaller than the corresponding versions of perceived burdensomeness subscales. Both perceived burdensomeness and thwarted belongingness subscales had significant correlation coefficients with the calibration standards of PSSS and UCLA Loneliness Scale. The result verified the views of Van Orden (2009) that interpersonal interactions characterized by low closeness or low frequency could not fully satisfy the sense 
of belonging, and might lead to feelings of loneliness and perceptions of insufficient social support. The criterion validities between the two subscales of each version were similar.

To examine concurrent predictive validity, the regression equations were conducted. Both perceived burdensomeness and thwarted belongingness of each version were significant predictors of acquired capability for suicide (see Table 4).

TABLE 4 | Regression models of perceived burdensomeness and thwarted belongingness predicting acquired capability for suicide.

\begin{tabular}{lccccc}
\hline \multicolumn{1}{r}{ Model } & & $\boldsymbol{R}^{\mathbf{2}}$ & $\boldsymbol{F}$ & $\boldsymbol{t}$ & $\boldsymbol{p}$ \\
\hline INQ-25 & PB & 0.018 & 16.284 & 4.035 & 0.000 \\
& TB & 0.029 & 26.942 & 5.191 & 0.000 \\
INQ-18 & PB & 0.014 & 13.132 & 3.624 & 0.000 \\
& TB & 0.029 & 26.833 & 5.180 & 0.000 \\
INQ-15 & PB & 0.026 & 23.746 & 4.873 & 0.000 \\
& TB & 0.029 & 26.833 & 5.180 & 0.000 \\
INQ-12 & PB & 0.017 & 15.624 & 3.953 & 0.000 \\
& TB & 0.023 & 20.848 & 4.566 & 0.000 \\
INQ-10 & PB & 0.029 & 26.650 & 5.162 & 0.000 \\
& TB & 0.032 & 30.176 & 5.493 & 0.000
\end{tabular}

\section{Differential Item Functioning}

To examine the differential item functioning of 25 items, the 'gender' variable divided into males and females was used to analyze. According to parameter comparison, the first item with the significance level of 0.01 and the 18th item with the significance level of 0.001 existed DIF (see Table 5). All versions contained the first item. Therefore, the versions without the 18 th item were suggested such as the 10 -item version and the 12 -item version.

\section{Average Test Information Curve}

To examine the average test information of each subscale, we calculated the total test information curve of each subscale presented in Figures 1, 2, and the average test information curves were presented in Figures 3, 4, which indicated the item information contained at each node along the $\theta$ scale. Measurement providing more information had higher reliability and more measurement precision. In Figure 3, the INQ-10 provided the most average test information at the range approximately from -0.8 to 2 standard deviations of perceived burdensomeness, among the five versions. At high ranges of $\theta$ value, the five scales provided a similar amount of information, while at other ranges of $\theta$ value, the INQ-10 provided the least in the five scales. On the whole, the five

TABLE 5 | DIF statistics for graded response model.

\begin{tabular}{|c|c|c|c|c|c|c|c|c|c|}
\hline Item & Total $X^{2}$ & d.f. & $p$ & $X_{\mathrm{a}}^{2}$ & d.f. & $p$ & $x_{\mathrm{cla}}^{2}$ & d.f. & $p$ \\
\hline 1 & 21.2 & 7 & 0.0035 & 2.6 & 1 & 0.1091 & 18.6 & 6 & $0.0049^{\star *}$ \\
\hline 2 & 9.5 & 7 & 0.2208 & 0.4 & 1 & 0.5193 & 9 & 6 & 0.1707 \\
\hline 3 & 12 & 7 & 0.0993 & 3.4 & 1 & 0.0666 & 8.7 & 6 & 0.1927 \\
\hline 4 & 5.1 & 7 & 0.6538 & 0 & 1 & 0.871 & 5 & 6 & 0.5409 \\
\hline 5 & 19.8 & 7 & 0.006 & 3.3 & 1 & 0.0681 & 16.5 & 6 & 0.0114 \\
\hline 8 & 5.8 & 7 & 0.5611 & 0.1 & 1 & 0.7006 & 5.7 & 6 & 0.4613 \\
\hline 9 & 4.5 & 7 & 0.7207 & 0.1 & 1 & 0.7507 & 4.4 & 6 & 0.623 \\
\hline 10 & 8.6 & 7 & 0.2839 & 4.1 & 1 & 0.0441 & 4.6 & 6 & 0.6029 \\
\hline 11 & 5.2 & 7 & 0.6381 & 0.4 & 1 & 0.5063 & 4.7 & 6 & 0.578 \\
\hline 12 & 3.9 & 7 & 0.7866 & 0.8 & 1 & 0.3662 & 3.1 & 6 & 0.7934 \\
\hline 16 & 10.3 & 7 & 0.1695 & 0.1 & 1 & 0.8068 & 10.3 & 6 & 0.1129 \\
\hline 17 & 6.4 & 7 & 0.4954 & 1.6 & 1 & 0.2029 & 4.8 & 6 & 0.5746 \\
\hline 18 & 26.9 & 7 & 0.0004 & 0.1 & 1 & 0.8133 & 26.8 & 6 & $0.0002^{\star \star \star}$ \\
\hline 19 & 16 & 7 & 0.0252 & 0.2 & 1 & 0.6267 & 15.8 & 6 & 0.0151 \\
\hline 20 & 14 & 7 & 0.0516 & 2.2 & 1 & 0.1416 & 11.8 & 6 & 0.0665 \\
\hline 21 & 20.1 & 7 & 0.0053 & 5.3 & 1 & 0.0214 & 14.8 & 6 & 0.0217 \\
\hline 22 & 13.3 & 7 & 0.0651 & 5.2 & 1 & 0.0227 & 8.1 & 6 & 0.2298 \\
\hline 23 & 15.9 & 7 & 0.0263 & 7.7 & 1 & 0.0056 & 8.2 & 6 & 0.2239 \\
\hline 24 & 14.3 & 7 & 0.0463 & 0.6 & 1 & 0.4339 & 13.7 & 6 & 0.0335 \\
\hline 25 & 10.6 & 7 & 0.1591 & 0.1 & 1 & 0.7263 & 10.4 & 6 & 0.1075 \\
\hline
\end{tabular}

Total $X^{2}$, total variance; $X^{2 a}$, within-group variance; $X_{c \mid a}^{2}$, between-group variance. ${ }^{* \star} p<0.01 .{ }^{\star \star *} p<0.001$. 


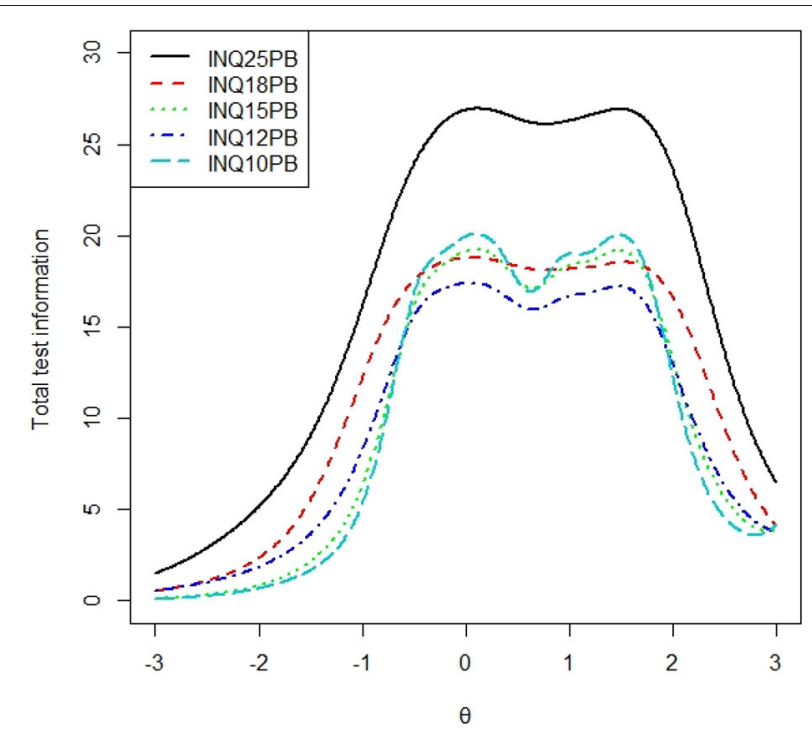

FIGURE 1 | Total test information curves of perceived burdensomeness of each version. INQ25PB, perceived burdensomeness subscale of INQ-25; INQ18PB, perceived burdensomeness subscale of INQ-18; INQ15PB, perceived burdensomeness subscale of INQ-15; INQ12PB, perceived burdensomeness subscale of INQ-12; INQ10PB, perceived burdensomeness subscale of INQ-10, same as below.

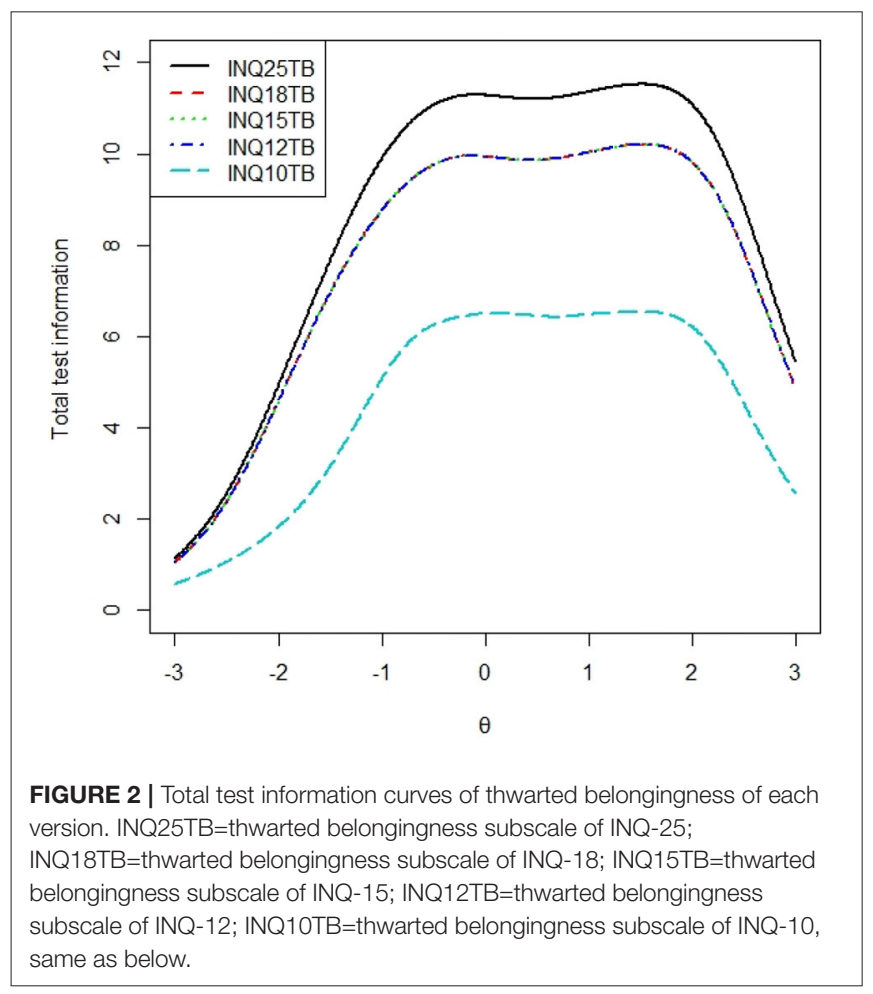

scales could provide proper average test information. The results suggested that the INQ-10 could provide more measurement precision for varying degrees of perceived burdensomeness, and this suggested that the INQ-10 might be more useful

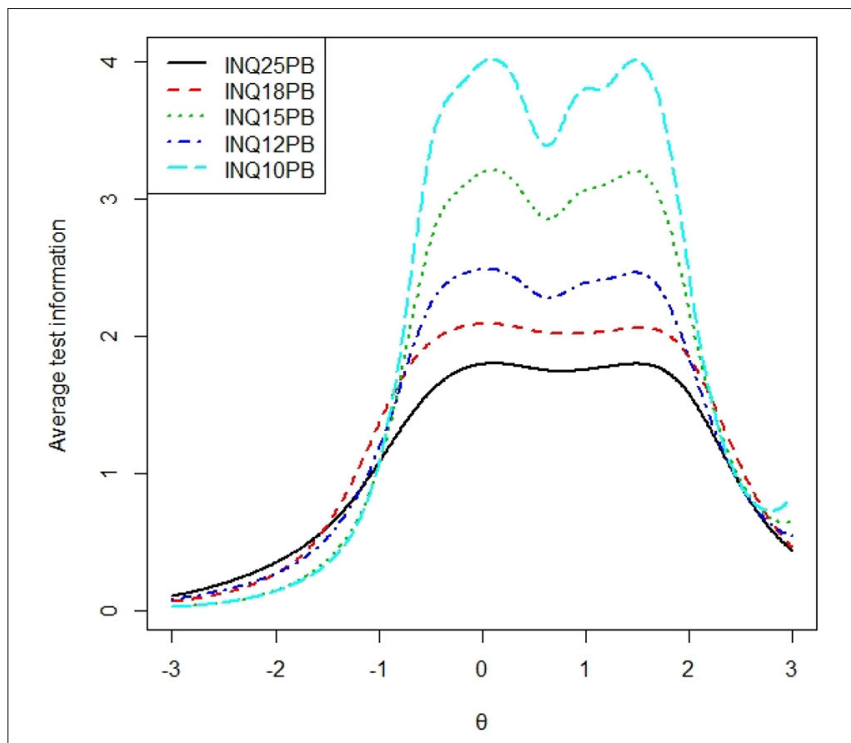

FIGURE 3 | Average test information curves of perceived burdensomeness of each version.

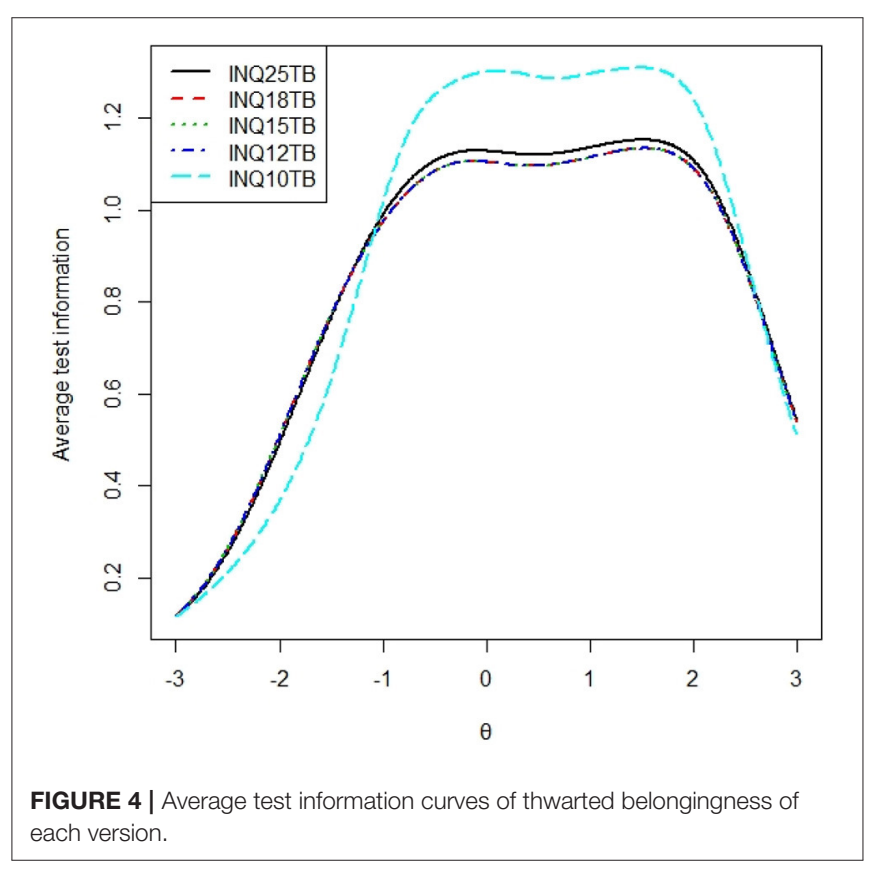

in teenage samples for measuring perceived burdensomeness in clinical trials and measuring perceived burdensomeness as an index of treatment response. In Figure 4, above -1 standard deviations of thwarted belongingness, the INQ-10 provided the most average test information, while at other ranges of $\theta$ value, the INQ-10 was the least in the five scales, but close to the other versions. Similar to the result of perceived burdensomeness subscales, the thwarted belongingness subscale of the INQ-10 could provide higher reliability and more measurement precision for varying degrees of thwarted 
TABLE 6 | Conversion table of five versions of INQ based on expected scores.

\begin{tabular}{|c|c|c|c|c|c|c|c|c|c|c|}
\hline \multirow[t]{2}{*}{$\theta$ Scale } & \multicolumn{5}{|c|}{ Perceived burdensomeness } & \multicolumn{5}{|c|}{ Thwarted belongingness } \\
\hline & INQ25 & INQ18 & INQ15 & INQ12 & INQ10 & INQ25 & INQ18 & INQ15 & INQ12 & INQ10 \\
\hline-3 & 15.23 & 9.04 & 6.00 & 7.04 & 5.00 & 10.06 & 9.05 & 9.05 & 5.04 & 5.02 \\
\hline-2.9 & 15.29 & 9.05 & 6.00 & 7.05 & 5.00 & 10.08 & 9.07 & 9.07 & 5.05 & 5.03 \\
\hline-2.8 & 15.37 & 9.06 & 6.00 & 7.06 & 5.00 & 10.10 & 9.10 & 9.10 & 5.07 & 5.04 \\
\hline-2.7 & 15.47 & 9.08 & 6.00 & 7.08 & 5.00 & 10.14 & 9.13 & 9.13 & 5.10 & 5.05 \\
\hline-2.6 & 15.59 & 9.11 & 6.00 & 7.11 & 5.00 & 10.19 & 9.18 & 9.18 & 5.14 & 5.07 \\
\hline-2.5 & 15.75 & 9.14 & 6.00 & 7.14 & 5.00 & 10.25 & 9.24 & 9.24 & 5.19 & 5.10 \\
\hline-2.4 & 15.94 & 9.19 & 6.00 & 7.19 & 5.00 & 10.34 & 9.33 & 9.33 & 5.25 & 5.14 \\
\hline-2.3 & 16.17 & 9.25 & 6.00 & 7.24 & 5.00 & 10.46 & 9.44 & 9.44 & 5.34 & 5.18 \\
\hline-2.2 & 16.45 & 9.32 & 6.01 & 7.32 & 5.00 & 10.61 & 9.58 & 9.58 & 5.46 & 5.25 \\
\hline-2.1 & 16.79 & 9.42 & 6.01 & 7.41 & 5.00 & 10.81 & 9.77 & 9.77 & 5.62 & 5.33 \\
\hline-2 & 17.19 & 9.53 & 6.01 & 7.52 & 5.01 & 11.06 & 10.01 & 10.01 & 5.81 & 5.44 \\
\hline-1.9 & 17.66 & 9.68 & 6.02 & 7.66 & 5.01 & 11.37 & 10.31 & 10.31 & 6.05 & 5.58 \\
\hline-1.8 & 18.20 & 9.85 & 6.03 & 7.83 & 5.02 & 11.76 & 10.67 & 10.67 & 6.35 & 5.75 \\
\hline-1.7 & 18.81 & 10.06 & 6.04 & 8.02 & 5.03 & 12.22 & 11.09 & 11.09 & 6.69 & 5.94 \\
\hline-1.6 & 19.50 & 10.31 & 6.06 & 8.24 & 5.04 & 12.75 & 11.58 & 11.58 & 7.08 & 6.17 \\
\hline-1.5 & 20.27 & 10.59 & 6.09 & 8.50 & 5.07 & 13.36 & 12.14 & 12.14 & 7.51 & 6.43 \\
\hline-1.4 & 21.11 & 10.92 & 6.14 & 8.78 & 5.11 & 14.06 & 12.77 & 12.77 & 7.99 & 6.72 \\
\hline-1.3 & 22.03 & 11.30 & 6.22 & 9.11 & 5.17 & 14.83 & 13.46 & 13.46 & 8.51 & 7.04 \\
\hline-1.2 & 23.04 & 11.76 & 6.33 & 9.48 & 5.26 & 15.68 & 14.22 & 14.22 & 9.06 & 7.41 \\
\hline-1.1 & 24.16 & 12.31 & 6.49 & 9.91 & 5.39 & 16.62 & 15.05 & 15.05 & 9.64 & 7.82 \\
\hline-1 & 25.40 & 12.96 & 6.71 & 10.41 & 5.57 & 17.62 & 15.94 & 15.94 & 10.24 & 8.28 \\
\hline-0.9 & 26.78 & 13.75 & 7.02 & 11.01 & 5.83 & 18.70 & 16.89 & 16.89 & 10.87 & 8.79 \\
\hline-0.8 & 28.32 & 14.68 & 7.43 & 11.73 & 6.17 & 19.83 & 17.90 & 17.90 & 11.50 & 9.34 \\
\hline-0.7 & 30.01 & 15.77 & 7.96 & 12.58 & 6.61 & 21.02 & 18.95 & 18.95 & 12.15 & 9.94 \\
\hline-0.6 & 31.87 & 17.01 & 8.62 & 13.55 & 7.16 & 22.26 & 20.06 & 20.06 & 12.79 & 10.57 \\
\hline-0.5 & 33.87 & 18.39 & 9.43 & 14.63 & 7.84 & 23.54 & 21.19 & 21.19 & 13.45 & 11.23 \\
\hline-0.4 & 36.00 & 19.87 & 10.36 & 15.79 & 8.62 & 24.85 & 22.36 & 22.36 & 14.11 & 11.92 \\
\hline-0.3 & 38.24 & 21.43 & 11.40 & 17.01 & 9.50 & 26.19 & 23.56 & 23.56 & 14.77 & 12.62 \\
\hline-0.2 & 40.58 & 23.06 & 12.55 & 18.28 & 10.48 & 27.54 & 24.77 & 24.77 & 15.42 & 13.35 \\
\hline-0.1 & 42.99 & 24.74 & 13.77 & 19.58 & 11.52 & 28.90 & 25.97 & 25.97 & 16.06 & 14.08 \\
\hline 0 & 45.45 & 26.45 & 15.05 & 20.91 & 12.61 & 30.24 & 27.17 & 27.17 & 16.68 & 14.82 \\
\hline-0.1 & 47.91 & 28.16 & 16.35 & 22.24 & 13.72 & 31.57 & 28.36 & 28.36 & 17.28 & 15.56 \\
\hline-0.2 & 50.35 & 29.85 & 17.66 & 23.55 & 14.84 & 32.88 & 29.52 & 29.52 & 17.85 & 16.29 \\
\hline-0.3 & 52.74 & 31.50 & 18.94 & 24.82 & 15.93 & 34.18 & 30.67 & 30.67 & 18.41 & 17.01 \\
\hline-0.4 & 55.06 & 33.07 & 20.18 & 26.03 & 16.98 & 35.46 & 31.81 & 31.81 & 18.95 & 17.72 \\
\hline-0.5 & 57.28 & 34.56 & 21.34 & 27.17 & 17.96 & 36.73 & 32.94 & 32.94 & 19.50 & 18.42 \\
\hline-0.6 & 59.40 & 35.96 & 22.41 & 28.24 & 18.87 & 38.00 & 34.07 & 34.07 & 20.05 & 19.13 \\
\hline-0.7 & 61.47 & 37.31 & 23.42 & 29.27 & 19.72 & 39.27 & 35.21 & 35.21 & 20.62 & 19.83 \\
\hline-0.8 & 63.53 & 38.67 & 24.40 & 30.32 & 20.55 & 40.57 & 36.36 & 36.36 & 21.21 & 20.54 \\
\hline-0.9 & 65.63 & 40.05 & 25.38 & 31.40 & 21.39 & 41.89 & 37.54 & 37.54 & 21.83 & 21.26 \\
\hline 1 & 67.79 & 41.47 & 26.41 & 32.52 & 22.28 & 43.24 & 38.75 & 38.75 & 22.47 & 21.99 \\
\hline 1.1 & 70.01 & 42.90 & 27.49 & 33.67 & 23.21 & 44.63 & 40.00 & 40.00 & 23.13 & 22.74 \\
\hline 1.2 & 72.31 & 44.36 & 28.61 & 34.84 & 24.19 & 46.05 & 41.27 & 41.27 & 23.82 & 23.50 \\
\hline 1.3 & 74.70 & 45.87 & 29.78 & 36.04 & 25.20 & 47.50 & 42.58 & 42.58 & 24.53 & 24.27 \\
\hline 1.4 & 77.19 & 47.45 & 31.00 & 37.31 & 26.26 & 48.99 & 43.92 & 43.92 & 25.26 & 25.06 \\
\hline 1.5 & 79.78 & 49.10 & 32.28 & 38.63 & 27.37 & 50.51 & 45.29 & 45.29 & 26.01 & 25.87 \\
\hline 1.6 & 82.43 & 50.78 & 33.59 & 39.97 & 28.51 & 52.05 & 46.68 & 46.68 & 26.78 & 26.69 \\
\hline 1.7 & 85.09 & 52.46 & 34.91 & 41.31 & 29.66 & 53.62 & 48.10 & 48.10 & 27.56 & 27.52 \\
\hline 1.8 & 87.71 & 54.10 & 36.19 & 42.60 & 30.75 & 55.19 & 49.53 & 49.53 & 28.35 & 28.35 \\
\hline 1.9 & 90.24 & 55.67 & 37.38 & 43.82 & 31.75 & 56.76 & 50.95 & 50.95 & 29.13 & 29.18 \\
\hline
\end{tabular}


TABLE 6 | Continued

\begin{tabular}{|c|c|c|c|c|c|c|c|c|c|c|}
\hline$\theta$ Scale & \multicolumn{5}{|c|}{ Perceived burdensomeness } & \multicolumn{5}{|c|}{ Thwarted belongingness } \\
\hline 2.1 & 94.72 & 58.39 & 39.31 & 45.87 & 33.31 & 59.81 & 53.72 & 53.72 & 30.66 & 30.76 \\
\hline 2.2 & 96.57 & 59.47 & 40.01 & 46.65 & 33.84 & 61.24 & 55.02 & 55.02 & 31.37 & 31.49 \\
\hline 2.3 & 98.15 & 60.34 & 40.56 & 47.26 & 34.22 & 62.56 & 56.21 & 56.21 & 32.02 & 32.15 \\
\hline 2.5 & 100.58 & 61.55 & 41.27 & 48.07 & 34.67 & 64.78 & 58.21 & 58.21 & 33.10 & 33.21 \\
\hline 2.6 & 101.48 & 61.94 & 41.49 & 48.32 & 34.79 & 65.67 & 59.01 & 59.01 & 33.52 & 33.62 \\
\hline 2.7 & 102.22 & 62.24 & 41.65 & 48.51 & 34.87 & 66.42 & 59.68 & 59.68 & 33.86 & 33.94 \\
\hline 2.8 & 102.81 & 62.45 & 41.76 & 48.64 & 34.92 & 67.04 & 60.24 & 60.24 & 34.13 & 34.20 \\
\hline 2.9 & 103.29 & 62.60 & 41.83 & 48.74 & 34.95 & 67.55 & 60.70 & 60.70 & 34.35 & 34.40 \\
\hline
\end{tabular}

belongingness. Overall, both the perceived burdensomeness subscale and the thwarted belongingness subscale of the INQ10 had the highest reliability and most measurement precision. Hence, in the five versions of INQ, choosing the INQ10 may be better in teenage samples at varying degrees of interpersonal needs.

\section{Expected Scores}

The expected scores of the five versions were calculated by transferring $\theta$ values based on GRM and presented in Table 6. The scores conversion means that the scores of the five versions measuring the same psychological trait can be compared with each other. The conversion table of the five scale scores provides help shifting one scale into another one and is useful for future study and application when different version scores need to be switched.

\section{DISCUSSION}

To date, the present study provides the first comparison of five versions of INQ simultaneously within teenage samples. Construct validity, internal consistency, validity, and average test information were compared for the five versions to (a) identify the version with the optimal overall psychometric characteristics in teenage samples to encourage future use, and (b) test the hypotheses of the IPTS and guide refinement of the IPTS.

Concerning validity, the INQ-15, INQ-12, and INQ-10 demonstrated adequate fit for a two-factor model for all global indices of fit, while the other longer versions did not. Thus, the INQ-15, INQ-12, and INQ-10 most consistently demonstrated construct validity, providing evidence in support of their continued use in teenage samples. The results of internal consistency and criterion validity showed that five versions of the INQ and their subscales all had good internal consistency. But the internal consistency coefficients of thwarted belongingness subscales were smaller than perceived burdensomeness subscales, which was similar to most previous researches (e.g., Bryan et al., 2010; Hill and Pettit, 2012; Monteith et al., 2013; Teo et al., 2018). The correlation coefficients of perceived burdensomeness and thwarted belongingness subscales were closed to each other. As for concurrent predictive validity, both perceived burdensomeness and thwarted belongingness of each version were significant predictors of acquired capability for suicide. The internal consistency, correlation coefficient, and concurrent validity of different versions did not provide any basis for recommending a specific version of the INQ. According to the DIF analysis, the first item with the significance level of 0.01 and 18th with the significance level of 0.001 item had DIF. All versions contained the first item. Therefore, the versions without the 18th item were suggested such as the 10 -item version and the 12 -item version. As for average test information, the INQ10 had clear advantages both in the perceived burdensomeness subscale and in thwarted belongingness subscale. In perceived burdensomeness subscales, the version that included more items provided less average test information. In thwarted belongingness subscales, the thwarted belongingness subscale of INQ-10 evidently provided the most average test information, and the thwarted belongingness subscale of INQ-25 provided the second most information. The average test information curves of thwarted belongingness subscales of the other three versions almost coincided. The thwarted belongingness subscales of INQ-18 and INQ-15 contained the same items, and the result demonstrated that 5 -item of thwarted belongingness subscale of the INQ-12 provided the same average test information with 9item of thwarted belongingness subscales of the INQ-15 and the INQ-18. Concerning the expected scores, the conversion of the five scale scores (see Table 6) enables the conversion of one scale shifting into another one. The conversion table can provide help for future studies and applications when one of the five scale scores needs to be transformed into another.

Overall, the result of average test information suggested the INQ-10 provided higher reliability and more measurement precision, and the 10 -item version with proper reliability and validity was demonstrated as an adequate fit for a twofactor model. Hence, the 10 -item version of INQ is the most suitable version for future use in teenage samples. In addition, the results above showed that perceived burdensomeness performed better in multiple indicators in comparison to 
thwarted belongingness. If different results are a consequence of measurement, it is necessary to take into consideration both theoretical and operational definitions of the thwarted belongingness and perceived burdensomeness. It is also possible that the thwarted belongingness subscale of the INQ is not adequately measuring the thwarted belongingness and developing a new self-report scale for thwarted belongingness is needed.

The results of this study should be viewed within the context of its limitations. First, the present study uses data from a teenage sample in China, which limits the generalizability of the results. According to the study, we cannot make a decision on which version of the INQ is best for the elderly and clinical samples. Furthermore, the INQ was demonstrated that it could predict suicidal ideation of the individual in previous studies (Joiner, 2005; Van Orden et al., 2010). But this study demonstrated that the INQ could predict the acquired capability for suicide and it was cross-sectional and did not examine the difference of predictive validity about suicidal ideation among the five versions, which can be done in the future. In addition, the five versions of INQ were derived from the response to INQ-25. Thus, current data did not take into account the possible influence of question order effects (e.g., consecutive questions might be answered more similarly than non-continuous questions). Furthermore, the internal consistency coefficients of thwarted belongingness subscales were smaller than the perceived burdensomeness subscales in this study. And findings in previous studies for the relationship between thwarted belongingness and suicidal ideation were weaker in comparison to perceived burdensomeness (Ma et al.,

\section{REFERENCES}

Anestis, M. D., and Joiner, T. E. (2011). Examining the role of emotion in suicidality: negative urgency as an amplifier of the relationship between components of the interpersonal psychological theory of suicidal behavior and lifetime number of suicide attempts. J. Affect. Disord. 129, 261-269. doi: 10.1016/j.jad.2010.08.006

Baertschi, M., Costanza, A., Richard-Lepouriel, H., Pompili, M., Sarasin, F., Weber, K., et al. (2017). The application of the interpersonal-psychological theory of suicide to a sample of Swiss patients attending a psychiatric emergency department for a non-lethal suicidal event. J. Affect. Disord. 210, 323-331. doi: 10.1016/j.jad.2016.12.049

Blumenthal, J. A., Burg, M. M., Barefoot, J., Williams, R. B., Haney, T., and Zimet, G. (1987). Social support, type a behavior, and coronary artery disease. Psychosom. Med. 49, 331-340. doi: 10.1097/00006842-198707000-00002

Brown, T. A. (2006). Confirmatory Factor Analysis for Applied Research. New York, NY: Guilford.

Browne, M. W., and Cudeck, R. (1992). Alternative ways of assessing model fit. Sociol. Methods Res. 21, 230-258. doi: 10.1177/0049124192021002005

Bryan, C. J. (2011). The clinical utility of a brief measure of perceived burdensomeness and thwarted belongingness for the detection of suicidal military personnel. J. Clin. Psychol. 67:981-92. doi: 10.1002/jclp.20726

Bryan, C. J., Clemans, T. A., and Hernandez, A. M. (2012). Perceived burdensomeness, fearlessness of death, and suicidality among deployed military personnel. Pers. Individ. Dif. 52, 374-379. doi: 10.1016/j.paid.2011.10.045

Bryan, C. J., Hernandez, A. M., Allison, S., and Clemans, T. (2013). Combat exposure and suicide risk in two samples of military personnel. J. Clin. Psychol. 69, 64-77. doi: 10.1002/jclp.21932
2016; Chu et al., 2017). In the future study, a new self-report scale for thwarted belongingness (TB) can be developed to expand the availability of valid measurement approaches for interpersonal risk.

\section{DATA AVAILABILITY STATEMENT}

The raw data supporting the conclusions of this article will be made available by the authors, without undue reservation.

\section{ETHICS STATEMENT}

The studies involving human participants were reviewed and approved by Ethics Committee of School of Psychology, Jiangxi Normal University. Written informed consent to participate in this study was provided by the participants' legal guardian/next of kin.

\section{AUTHOR CONTRIBUTIONS}

DT, XY, and YC selected the topic and made some modifications of the paper. JQ collected the data and wrote the manuscript. All authors contributed to the article and approved the submitted version.

\section{SUPPLEMENTARY MATERIAL}

The Supplementary Material for this article can be found online at: https://www.frontiersin.org/articles/10.3389/fpsyg. 2021.676361/full\#supplementary-material
Bryan, C. J., Morrow, C., Anestis, M., and Joiner, T. (2010). A preliminary test of the interpersonal-psychological theory of suicidal behavior in a military sample. Pers. Individ. Dif. 48, 347-350. doi: 10.1016/j.paid.2009.10.023

Carballo, J. J., Llorente, C., Kehrmann, L., Flamarique, I., Zuddas, A., and Arango, C. (2019). Psychosocial risk factors for suicidality in children and adolescents. Eur. Child Adolesc. Psychiatry 29, 759-776. doi: 10.1007/s00787-01801270-9

Centers for Disease Control and Prevention (2017). Available online at: http:// www.cdc.gov/violenceprevention/pub/youth_suicide.html (Retrieved March 30, 2020).

Chalmers, R. P. (2012). mirt: a multidimensional item response theory package for the r environment. J. Statisti. Softw. 48, 1-29. doi: 10.18637/jss.v048.i06

Chu, C., Buchman-Schmitt, J. M., Hom, M. A., Stanley, I. H., and Joiner, T. E. (2016). A test of the interpersonal theory of suicide in a large sample of current firefighters. Psychiatry Res. 240, 26-33. doi: 10.1016/j.psychres.2016.03.041

Chu, C., Buchman-Schmitt, J. M., Stanley, I. H., Hom, M. A., Tucker, R. P., Hagan, C. R., et al. (2017). The interpersonal theory of suicide: a systematic review and meta-analysis of a decade of cross-national research. Psychol. Bull. 143, 1313-1345. doi: 10.1037/bul0000123

Cukrowicz, K. C., Jahn, D. R., Graham, R. D., Poindexter, E. K., and Williams, R. B. (2013). Suicide risk in older adults: evaluating models of risk and predicting excess zeros in a primary care sample. J. Abnorm. Psychol. 122, 1021-1030. doi: $10.1037 /$ a0034953

Davidson, C. L., Wingate, L. R., Grant, D. M., Judah, M. R., and Mills, A. C. (2011). Interpersonal suicide risk and ideation the influence of depression and social anxiety. J. Soc. Clin. Psychol. 30, 842-855. doi: 10.1521/jscp.2011.30.8.842

Davidson, C. L., Wingate, L. R., Rasmussen, K. A., and Slish, M. L. (2009). Hope as a predictor of interpersonal suicide risk. Suicide Life-Threatening Behav. 39, 499-507. doi: 10.1521/suli.2009.39.5.499 
Fayers, P. (2004). Item response theory for psychologists. Q. Life Res. 13, 715-716. doi: 10.1023/B:QURE.0000021503.45367.f2

Fink-Miller, E. L. (2015). An examination of the interpersonal psychological theory of suicidal behavior in physicians. Suicide Life-Threat. Behav. 45, 488-494. doi: $10.1111 /$ sltb. 12147

Freedenthal, S., Lamis, D. A., Osman, A., Kahlo, D., and Gutierrez, P. M. (2011). Evaluation of the psychometric properties of the interpersonal needs questionnaire-12 in samples of men and women. J. Clin. Psychol. 67, 609-623. doi: $10.1002 /$ jclp. 20782

Hagan, C. R., Podlogar, M. C., Chu, C., and Joiner, T. E. (2015). Testing the interpersonal theory of suicide: the moderating role of hopelessness. Int. J. Cogn. Therapy 8, 99-113. doi: 10.1521/ijct.2015.8.2.99

Hallensleben, N., Spangenberg, L., Kapusta, N. D., Forkmann, T., and Glaesmer, H. (2016). The German version of the Interpersonal Needs Questionnaire (INQ) dimensionality, psychometric properties and population-based norms. J. Affect. Disord. 195, 191-198. doi: 10.1016/j.jad.2016.01.045

Hill, R. M., and Pettit, J. W. (2012). Suicidal ideation and sexual orientation in college students: the roles of perceived burdensomeness, thwarted belongingness, and perceived rejection due to sexual orientation. Suicide LifeThreat. Behav. 42, 567-579. doi: 10.1111/j.1943-278X.2012.00113.x

Hill, R. M., and Pettit, J. W. (2013). The role of autonomy needs in suicidal ideation: integrating the interpersonal-psychological theory of suicide and self-determination theory. Arch. Suicide Res. 17, 288-301. doi: $10.1080 / 13811118.2013 .777001$

Hill, R. M., Rey, Y., Marin, C. E., Sharp, C., Green, K. L., and Pettit, J. W. (2015). Evaluating the interpersonal needs questionnaire: comparison of the reliability, factor structure, and predictive validity across five versions. Suicide Life. Threat. Behav. 45, 302-314. doi: 10.1111/sltb.12129

Hu, L.-T., and Bentler, P. M. (1999). Cutoff criteria for fit indexes in covariance structure analysis: conventional criteria versus new alternatives. Struct. Eq. Model. 6, 1-55. doi: 10.1080/10705519909540118

Hunsley, J., and Mash, E. J. (2008). A Guide to Assessments That Work. Oxford: Oxford University Press. doi: 10.1093/med:psych/9780195310641.001.0001

Joiner, T. E. (2005). Why People Die by Suicide. Cambridge, MA: Harvard University Press.

Joiner, T. E., Van Orden, K. A., Witte, T. K., and Rudd, M. D. (2009). The Interpersonal Theory of Suicide: Guidance for Working With Suicidal Clients. Arlington, VA: American Psychiatric Association. doi: 10.1037/11869-000

Kim, M. (2001). Detecting DIF across the different language groups in a speaking test. Lang. Testing 18, 88-114. doi: 10.1177/026553220101800104

Klonsky, E. D., and May, A. M. (2014). Differentiating suicide attempters from suicide ideators: a critical frontier for suicidology research. Suicide Life-Threat. Behav. 44, 1-5. doi: 10.1111/sltb.12068

Klonsky, E. D., May, A. M., and Saffer, B. Y. (2016). Suicide, suicide attempts, and suicidal ideation. Ann. Rev. Clin. Psychol. 12, 307-330. doi: 10.1146/annurev-clinpsy-021815-093204

Lamis, D. A., and Lester, D. (2012). Risk factors for suicidal ideation among African American and European American college women. Psychol. Women Q. 36, 337-349. doi: $10.1177 / 0361684312439186$

Lamis, D. A., and Malone, P. S. (2011). Alcohol-related problems and risk of suicide among college students: the mediating roles of belongingness and burdensomeness. Suicide Life-Threat. Behav. 41, 543-553. doi: 10.1111/j.1943-278X.2011.00052.x

Li, X. M., Xin, T. G., Yuan, J., Lv, L. X., Tao, J. L., and Liu, Y. (2015). Validity and reliability of the interpersonal needs questionnaire in Chinese college students. Chin. J. Clin. Psychol. 23, 590-593. doi: 10.16128/j.cnki.1005-3611.2015.04.005

Lord, F. M. (1977). A broad-range tailored test of verbal ability. Appl. Psychol. Measure. 1, 95-100. doi: 10.1177/014662167700100115

Ma, J., Batterham, P. J., Calear, A. L., and Han, J. (2016). A systematic review of the predictions of the interpersonal-psychological theory of suicidal behavior. Clin. Psychol. Rev. 46, 34-45. doi: 10.1016/j.cpr.2016.04.008

Mandracchia, J. T., and Smith, P. N. (2015). The interpersonal theory of suicide applied to male prisoners. Suicide Life-Threat. Behav. 45, 293-301. doi: $10.1111 /$ sltb.12132

Monteith, L. L., Menefee, D. S., Pettit, J. W., Leopoulos, W. L., and Vincent, J. P. (2013). Examining the interpersonal-psychological theory of suicide in an inpatient veteran sample. Suicide Life-Threat. Behav. 43, 418-428. doi: $10.1111 /$ sltb.12027
Olino, T. M., Yu, L., Klein, D. N., Rohde, P., Seeley, J. R., Pilkonis, P. A., et al. (2012). Measuring depression using item response theory: an examination of three measures of depressive symptomatology. Int. J. Methods Psychiatr. Res. 21, 76-85. doi: 10.1002/mpr.1348

Parellada, M., Saiz, P., Moreno, D., Vidal, J., Llorente, C., Alvarez, M., et al., (2008). Is attempted suicide different in adolescent and adults? Psychiatry Res. 157, 131-137. doi: 10.1016/j.psychres.2007.02.012

Rasmussen, K. A., and Wingate, L. R. (2011). The role of optimism in the interpersonal-psychological theory of suicidal behavior. Suicide Life. Threat. Behav. 41, 137-148. doi: 10.1111/j.1943-278X.2011.00022.x

Russell, D., Peplau, L. A., and Cutrona, C. E. (1980). The revised UCLA loneliness scale: concurrent and discriminant validity evidence. J. Pers. Soc. Psychol. 39, 472-480. doi: 10.1037/0022-3514.39.3.472

Samejima, F. (1969). Estimation of latent ability using a response pattern of graded scores. Psychometrika 34, 1-97. doi: 10.1007/BF03372160

Silva, C., Chu, C., Monahan, K. R.,., and Joiner, T. E. (2015). Suicide risk among sexual minority college students: a mediated moderation model of sex and perceived burdensomeness. Psychol. Sex. Orient. Gender Diversity 2, 22-33. doi: $10.1037 / \mathrm{sgd} 0000086$

Suh, S., Ebesutani, C. K., Hagan, C. R., Rogers, M. L., Hom, M. A., Ringer, F. B., et al. (2017). Cross-cultural relevance of the interpersonal theory of suicide across Korean and U.S. undergraduate students. Psychiatry Res. 251, 244-252. doi: 10.1016/j.psychres.2017.02.005

Teo, D. C. H., Suárez, L., and Oei, T. P. S. (2018). Validation of the interpersonal needs questionnaire of young male adults in Singapore. PLos ONE 13, 1-9. doi: 10.1371/journal.pone.0198839

Van Orden, K. A. (2009). Construct Validity of the Interpersonal Needs Questionnaire. (Unpublished doctoral dissertation), Florida State University, Florida, United States.

Van Orden, K. A., Cukrowicz, K. C., Witte, T. K., and Joiner, T. E. (2012). Thwarted belongingness and perceived burdensomeness: construct validity and psychometric properties of the interpersonal needs questionnaire. Psychol. Assess. 24, 197-215. doi: 10.1037/a0025358

Van Orden, K. A., Witte, T. K., Cukrowicz, K. C., Braithwaite, S. R., Selby, E. A., and Joiner, T. E. (2010). The interpersonal theory of suicide. Psychol. Rev. 117, 575-600. doi: 10.1037/a0018697

Van Orden, K. A., Witte, T. K., Gordon, K. H., Bender, T. W., and Joiner, T. E. (2008a). Suicidal desire and the capability for suicide: tests of the interpersonalpsychological theory of suicidal behavior among adults. J. Consult. Clin. Psychol. 76, 72-83. doi: 10.1037/0022-006X.76.1.72

Van Orden, K. A., Witte, T. K., James, L. M., Castro, Y., Gordon, K. H., Braithwaite, S. R., et al. (2008b). Suicidal ideation in college students varies across semesters: the mediating role of belongingness. Suicide Life-Threat. Behav. 38, 427-435. doi: 10.1521/suli.2008.38.4.427

Wong, Y. J., Koo, K., Tran, K. K., Chiu, Y.-C., and Mok, Y. (2011). Asian American college students' suicide ideation: a mixed-methods study. J. Couns. Psychol. 58, 197-209. doi: 10.1037/a0023040

World Health Organization (2019). Newsroom-Fact sheets. Available online at: https://www.who.int/news-room/fact-sheets/detail/suicide (Retrieved March 30, 2020).

Yang, L., Pan, D., Li, Z., and Liu, X. (2019). Validation of a Chinese version of the acquired capability for suicide scale. Death Stud. 3, 1-8. doi: 10.1080/07481187.2019.1671545

Zhang, J., Lester, D., Zhao, S., and Zhou, C. (2013). Suicidal ideation and its correlates: testing the interpersonal theory of suicide in chinese students. Arch. Suicide Res. 17, 236-241. doi: 10.1080/13811118.2013.805643

Conflict of Interest: The authors declare that the research was conducted in the absence of any commercial or financial relationships that could be construed as a potential conflict of interest.

Copyright (c) 2021 Quan, Yu, Cai and Tu. This is an open-access article distributed under the terms of the Creative Commons Attribution License (CC BY). The use, distribution or reproduction in other forums is permitted, provided the original author(s) and the copyright owner(s) are credited and that the original publication in this journal is cited, in accordance with accepted academic practice. No use, distribution or reproduction is permitted which does not comply with these terms. 